\title{
球磨过程中介质群运动区域划分方法研究*
}

\author{
梁 曼 ${ }^{1,2}$ 孙卫红 ${ }^{1}$ 孙 毅 ${ }^{2}$ 项京成 ${ }^{2}$ \\ (1. 中国计量大学机电工程学院 杭州 310018;
}

2. 浙江工业大学先进加工技术教育部重点实验室＼cjkstart杭州３10014)

\begin{abstract}
摘要: 球磨过程中介质群运动状态变化对物料的破碎效率影响极大, 通过对介质群运动状态进行区域划分, 探究不同球磨工 况下的介质群运动区域特征更能有效揭示介质群对物料的破碎方式和有效破碎区域。针对理论划分介质群运动区域过于理想、 单一, 试验追踪法成本过高的问题, 提出了一种介质群运动区域划分的新方法一EDEM 网格划分法。首先把筒体的横截面划 分为若干个微元, 利用数理统计的方法得到介质群位置概率分布函数, 获取介质群运动速度区域分布图和碰撞特性区域分布 图。然后给出了回转运动、螺旋运动及复合颤振运动三种工况下介质群运动区域的划分实例, 并与试验追踪法对比, 验证了 EDEM 网格划分法的准确性和有效性。最后通过粉磨试验中进料的破碎速率和微细颗粒产率探讨了介质群运动区域特性对颗粒的破碎 方式和有效破碎区域的影响效果。本研究为优化球磨过程的影响因素和操作参数提供了一种快捷、有效的方法。
\end{abstract}

关键词: 介质群运动状态; EDEM 网格划分法; 速度/碰撞特性区域; 破碎率; 微细颗粒产率

中图分类号: TD453

\section{Study on the Method of Dividing Media Motion Region in Ball Milling}

\author{
LIANG Man $^{1,2}$ SUN Weihong ${ }^{1}$ SUN Yi ${ }^{2}$ XIANG Jingcheng ${ }^{2}$ \\ (1. College of Mechanical and Electrical Engineering, China Jiliang University, Hangzhou 310018; \\ 2. Key Laboratory of Advanced Processing Technology of Ministry of Education, Zhejiang University of \\ Technology, Hangzhou 310014)
}

\begin{abstract}
The motion morphology of grinding media has an important effect on particles breakage efficiency in ball milling. In order to reveal the broken way and effective broken region when the motion morphology changes in different milling conditions, research on the regional division of the motion morphology of grinding media has been conducted. The theoretical dividing method is too ideal and less application, while the experimental tracking method costs too much time and money. Therefore, a new method of dividing the media motion morphology regions using EDEM grid partition has been proposed. The location function for each grid which is divided from the cross-section of the mill is obtained by the numerical statistics, combined with the regional distributions of media velocity vectors and collision characteristics, the regional distribution examples in three different milling condition, including rotational motion, spiral motion and coupled flutter motion have been provided. The comparison result with the experimental tracking method has verified the validity and availability of the EDEM grid partition method. In the end, the grinding test has been conducted, and the particles' broken way and effective broken region imposed by the media motion morphology has been discussed according to the particles broken effect and broken rate. This conclusion provides a fast and effective method to study and optimize the region changes in different influence factors.
\end{abstract}

Key words: grinding media motion morphology; EDEM grid partition method; particles' velocity and collision region; crashed rate; productivity of fine particles

\section{0 前言}

球磨过程中离散介质群对物料的破碎作用主要 有法向冲击和切向研磨, 不同工况下的作用区域差

* 国家自然科学基金资助项目(51675484 和 51275474)。20191219 收到初 稿, 20200525 收到修改稿
异很大 ${ }^{[1]}$ 。不同作用区域的位置分布和区域范围直 接关系到磨筒内物料的破碎方式和破碎效率 ${ }^{[2]}$ 。同 时，不同运动区域中介质群之间的碰撞特性和碰撞 能级大小对物料的破碎作用也存在显著差异。因此, 通过对介质群运动区域划分, 分析介质群在磨筒中 的运动区域分布以及碰撞特性的区域分布，对研究 球磨过程中物料的有效破碎区域和作用机理具有指 导意义。影响介质群和物料运动状态的因素众多, 
比如磨机结构、筒体运动模式、球磨过程工作参数 等 ${ }^{[3-5]}$, 其中区域分布的差异性主要取决于介质群和 物料的运动状态。国内外学者对磨机筒内介质群运 动状态及其影响因素做了大量的研究工作 ${ }^{[6-8]}$, 大多 是改进试验设备或增设状态采集装置, 如用高速摄 像机采集介质群运动图像 ${ }^{[9]}$; 或在筒体内壁安装传 感器, 通过检测介质群对筒壁的冲击力分析不同影 响因素(填充率、介质尺寸等)下介质群的运动状态 ${ }^{[10]}$; 刘军德等[11]通过设计与研磨介质体积、质量等效, 且 带有加速度传感器的检测球获取磨筒内介质群的运 动状态及轨迹。一个稳定周期内, 磨筒内介质群和 物料在运动形态可划分为四个区域: 提升区域; 泻 落(滑移)区域; 抛落区域和加速区域 ${ }^{[12]}$ 。在球磨初、 中期阶段, 由于磨介和物料的运动状态基本一致, 且在运动过程中混合均匀, 因此把磨介和物料的混 合物认为是密度均匀的 “介质” [13]。笔者曾对磨筒 内介质的运动状态开展理论研究, 得到了介质的脱 离角表达式及降落区域方程 ${ }^{[14]}$ 。樊狄峰等 ${ }^{[15]}$ 分析了 球磨过程中泻落、抛落以及两者混合运动状态下的 介质运动状态, 给出了各个运动区域的介质群数量 表达式。POWELL 等[16]通过双平面 X 射线追踪磨 筒中单个介质的运动轨迹, 分析了介质群的整体运 动形态, 并定义了介质群的区域结构参数。

MCBRIDE 等[17]在 POWELL 的基础上阐述了不同 区域的含义, 给出了介质群循环中心和运动平衡面 两个运动形态特征。为了识别循环中心和平衡面, 需要人工观察介质群运动图片及分析 $\mathrm{X}$ 射线追踪的 单个介质的运动轨迹, 然而该方法存在一定的主观 偏差。GOVENDER 等 ${ }^{[18]}$ 利用数理统计法得到了磨 筒内介质群位置概率分布函数, 并对介质群的运动形态 进行了区域划分, 但划分步骤繁琐, 对不同影响因素中 的介质群运动区域特性的研究不具有普适性。

因磨筒内部介质群运动情况复杂、环境恶劣, 高速摄像或轨迹追踪的试验方法很难快速准确地反 应介质群的运动及碰撞特性, 对不同影响因素下介 质群运动区域变化的反应也不够灵敏, 且试验研究 的时间成本高, 耗资大。近几年离散单元法(Discrete element method, DEM)模拟介质群的运动过程受到 国内外学者的广泛关注 ${ }^{[19-21]}$, 较多学者利用 DEM 实现了对球磨过程中介质群运动状态、接触作用以 及碰撞特性的仿真模拟, 并通过大量的对比试验验 证了离散元模拟与实际情况的吻合程度 ${ }^{[22]}$, 如利用 DEM 模拟介质群与筒壁的碰撞过程, 记录介质群与 筒壁之间的碰撞次数, 与同等条件下的测试磨机的 筒壁声信号进行比较, 发现两者吻合性较好 ${ }^{[23]}$; 或
在双平面 X 射线的试验装置中获取的介质群运动轨 迹曲线, 无论是沿筒体径向还是轴向, 与通过 DEM 仿真中得到的介质群运动轨迹线都基本吻合 ${ }^{[17]}$ 。

本文在对比分析了理论划分方法和试验追踪方 法的基础上, 针对现有球磨过程中介质群运动区域 划分方法的不足, 提出了一种基于离散元 DEM 的 介质群运动区域划分方法。通过 EDEM 数值模型获 取介质群在一个或多个稳定运动周期中的位置概率 分布函数, 利用 Matlab 对每个网格中的离散数据进 行编程解析, 基于数理统计与概率论构建介质群各 运动参量与位置区域之间的关联性, 获取介质群运 动特征参数区域分布图。结合不同运动模式(回转运动、 螺旋运动和复合颤振运动)下介质群运动区域划分实 例及粉磨试验结果, 探讨介质群运动区域分布特征对 物料的破碎速率和微细颗粒产率的影响效果。

\section{1 介质群运动区域划分方法}

\section{1 理论划分方法}

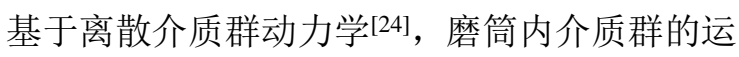
动轨迹及速度变化梯度在不同区域有明显差异, 对 磨筒内介质群运动状态的区域划分是提高球磨过程 破碎效率的前提条件。稳定运动周期内, 介质群的 循环运动过程呈动态平衡且运动形态基本上呈周期 性变化 ${ }^{[25]}$ 。孙毅等 ${ }^{[26]}$ 将磨筒内介质群的运动区域划 分为剪切区、冲击区和乏能区, 如图 1 所示, 并分 析了颤振耦合的运动模式下各区域边界的曲线方程。 本文依据磨筒中最外层介质的受力状态, 以四边形 角螺旋衬板为例, 开展了最外层介质运动区域的理 论划分研究 ${ }^{[14,27]}$, 划分结果如图 2 所示。

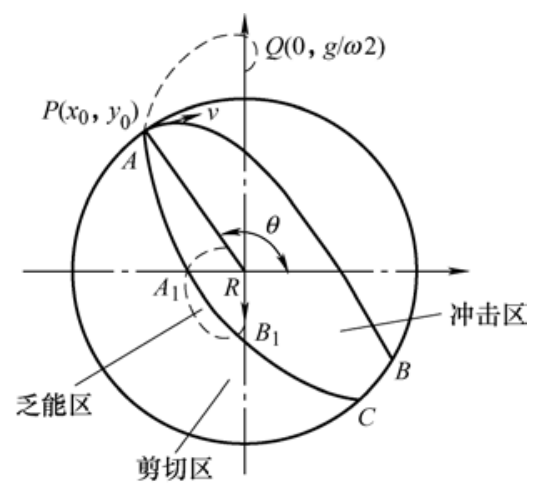

图 1 磨筒内介质群运动区域理论划分示意图

上述理论划分结果表明不同区域的介质群运动 特征对物料的作用区域差异性较明显。但仅能识别 各区域的边界曲线或降落位置, 区域中介质群的运 动特征及区域范围都无法定量统计, 不能及时地响 应磨筒运动状态的变化, 适用范围较小。 


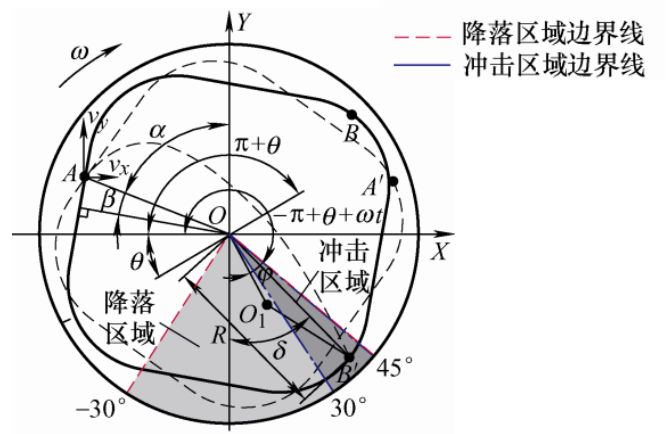

图 2 介质从 $A$ 点抛落至降落点 $B$ 的运动轨迹及区域边界线

\section{2 试验追踪法}

MCBRIDE 等[17]把封闭筒体中充分混合的介质 群和物料视作一个质量守恒系统, 忽略球磨过程中 的质量磨损, 混合物运动可近似看作颗粒流动体, 介质群看作质量流。如图 3 所示, 把磨筒的横截面 划分为若干组水平控制面和垂直控制面。通过试验 追踪装置记录并统计介质群轨迹线, 通过每个垂直
控制面的数量、交点的位置以及交点的方向，得到 通过每个垂直控制面的质量流数量以及平衡点，利 用质量流平衡点的位置标记该控制面组的质量流水

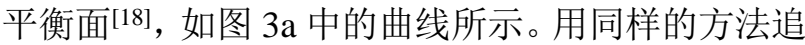
踪记录水平控制面上的质量流平衡点, 可得到质量 流垂直平衡面，两平衡面的交点即是质量流循环中 心(CoC), 如图 3b 所示。循环中心点以上实线部分 与中心点以下实线部分构成了整个介质群的运动平 衡曲面, 如图 3b 中的黑色虚线所示 ${ }^{[18]}$ 。

与理论划分方法相比，试验追踪法划分的运动 区域更具体、更准确地表达了磨筒中介质群的实际 运动状态，直观地反映介质群的流向以及不同运动 区域分界面的位置，且对不同工况中的介质群运动 状态的适用性都较好。但其局限性在于: 需要高成 本的精密追踪设备来追踪和记录介质群的运动轨迹, 对设备要求高, 划分过程较复杂。

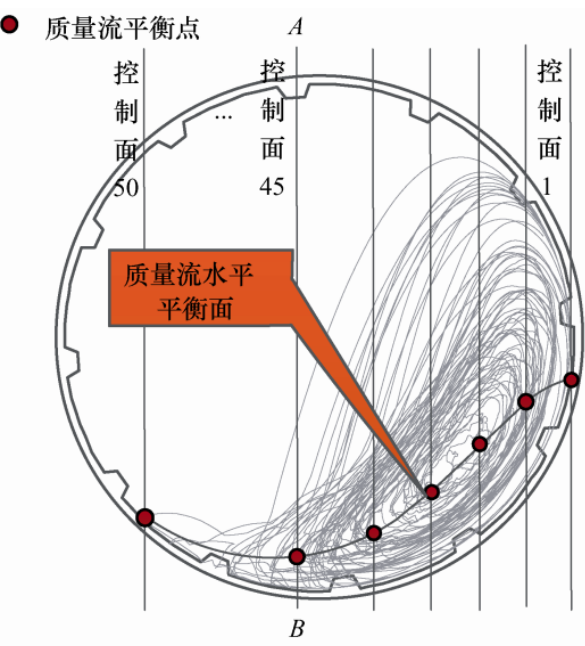

(a) 垂直控制面组以及由垂直控制面得 到的质量流水平平衡面

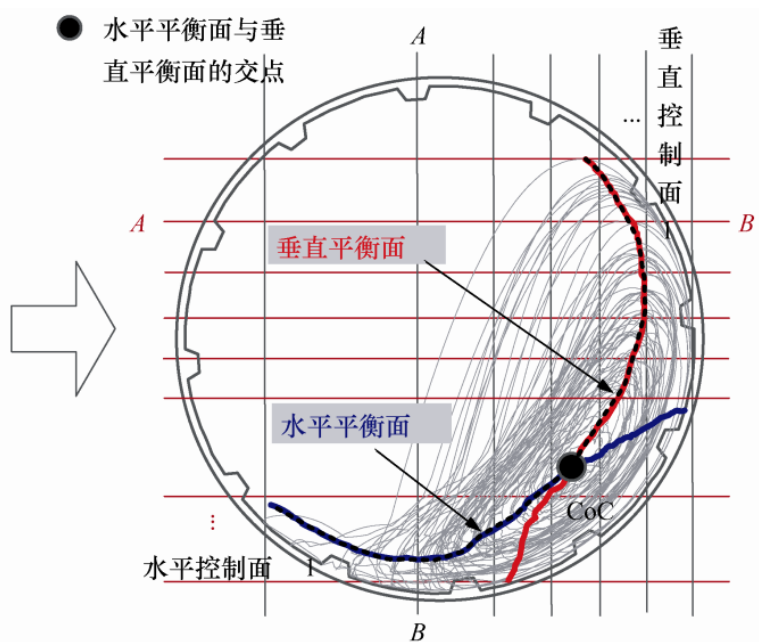

(b) 由垂直控制面和水平控制面得到的 介质群运动平衡面

图 3 试验追踪法划分磨筒内介质群运动平衡面

\subsection{EDEM 网格划分法}

试验追踪法对磨筒中的区域划分结果显示：水 平控制面组( $Y$ 向)中 $A \rightarrow B$ 向的介质群数量和 $B \rightarrow A$ 向的数量相等的位置点, 即图 3a 中的交点是水平方 向质量流平衡点, 这些平衡点构成了质量流垂直平 衡面, 同时也是介质群上升和下落( $Z$ 向)运动的分界 面。此时介质群 $Z$ 向速度接近零值。同理, 由垂直 控制面组( $Z$ 向)划分的介质流水平平衡面是介质群 水平运动 ( $Y$ 向)的分界面, 其速度也近似零值。根据 介质群的这种运动速度矢量特征, 利用 EDEM 构建 磨筒中介质群运动的仿真模型。介质群在不同区域 的位置分布示意图如图 4 所示。由于介质群运动状 态的瞬时性, 需要选取一个或者多个稳定的运动周
期获取介质群完整的运动形态特征。

网格划分法是基于数理统计与概率论构建各物 理参量与位置区域之间的关联性。首先，在 EDEM 的后处理模块对筒体几何模型进行网格划分，按照 直角坐标 $Z-Y$ 把磨筒的横截面划分为 $n \times n$ 个网格 $(n$ 值取决于介质尺寸), 介质群出现在每个网格中的概 率是随机的, 假设 $P$ 是概率测度, $x$ 是随机变量, 则介质出现在任何一个网格的概率 [28]

$$
F(x)=P\left(x_{1} \leqslant X \leqslant x_{2}\right)(x \in R)
$$

每个网格的位置坐标已知, 统计介质在周期运动中出 现在每个网格中的频率, $F(x)$ 在 $x$ 处的函数值即是 $X$ 落在一个网格区间 $\left[x_{1}, x_{2}\right]$ 上的概率值。 $X$ 的取值只有 $x_{1}<x_{2}<\cdots<x_{n}$, 则分布函数 $F(x)$ 的值域是离散的 


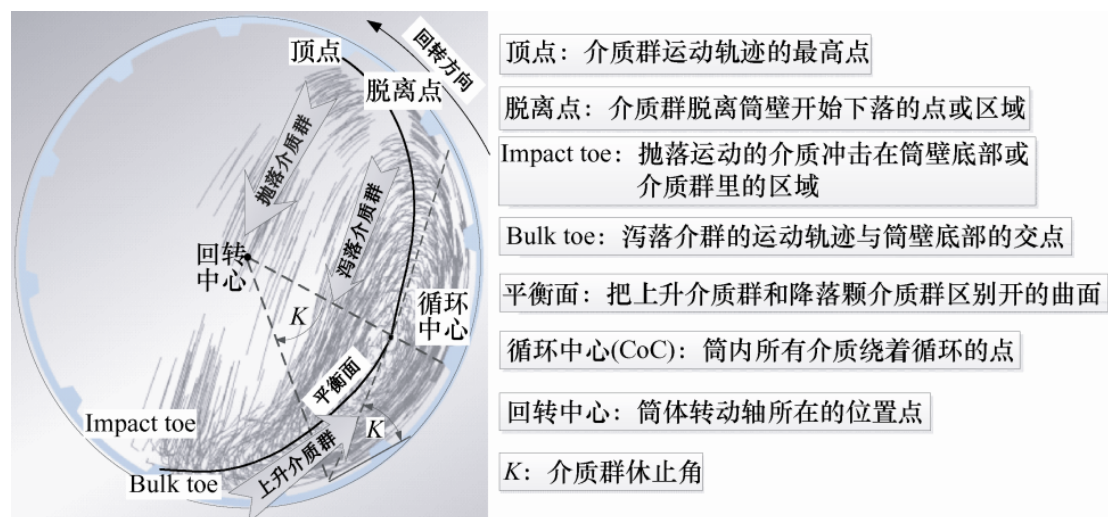

图 4 EDEM 模型中介质群运动形态与各区域定义及分布示意图

$$
F_{X}\left(x_{i}\right)=\sum_{j=1}^{i} P\left(x_{j}\right)
$$

且有

$$
\sum_{k=1}^{n} P\left(x_{i}\right)=1
$$

已知介质群在每个网格的概率分布函数 $F_{X}\left(x_{i}\right)$ 和网格对应的位置坐标可以得到介质群在 整个筒体横截面上的分布区域, 另从 EDEM 仿真 结果中能够导出对应网格坐标的介质群的运动

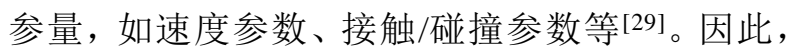
以一个或多个稳定运动周期的运动参量为对象, 对应介质群的运动区域分布, 通过 Matlab 编程解 析即可得到磨筒内介质群不同运动参量的区域 分布, 称为 EDEM 网格划分法。最后, 用不同颜 色等级代表介质群运动参数变化梯度, 绘制出不 同运动参数的区域分布图, 颜色等级接近零值的 区域即是介质群运动平衡面。EDEM 网格划分法 的具体操作流程如图 5 所示。

\section{2 介质群运动特性区域划分过程}

\section{1 速度矢量区域分布}

以回转运动中介质群的运动形态为例, 利用 EDEM 网格划分法对介质群的运动速度分布进行区 域划分, 得到的速度矢量分布区域如图 6 所示, 介 质群的速度梯度变化由不同灰度等级表征。图 6a、 $6 \mathrm{~b}$ 分别给出了介质群在垂直和水平方向的速度矢 量分布区域和质量流垂直和水平平衡曲面, 图 6c 中把两条平衡曲面分段地结合在一起得到介质群运 动平衡面, 该平衡面与通过试验追踪法获取的平衡 面(图 6c 中实线所示)几乎重合, 验证了 EDEM 网格 划分法的准确性和有效性。由于基于 EDEM 网格模

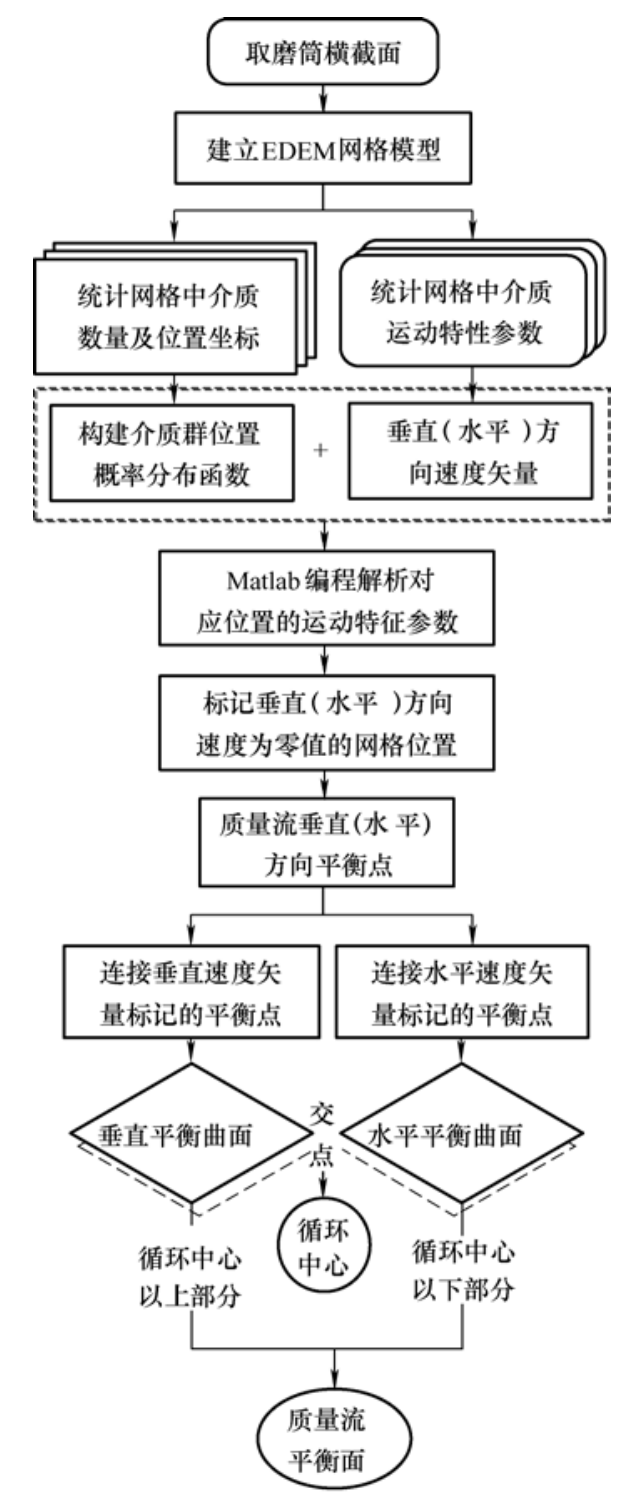

图 5 EDEM 网格划分法的划分流程图

型的介质群速度矢量区域划分过程与球磨过程的工 况变化无关, 划分方法不受粉磨条件影响, 对不同工 况、不同属性参数的介质群运动区域划分具有普适性。

图 6a 中可以直观地看出介质流垂直平衡面把 磨筒中的介质群运动区域划分为两个范围差距比较 
明显的区域: 上升的介质群运动区域范围较小, 速 度梯度变化较小; 下落的介质群运动区域范围大, 且速度梯度变化大。同样地, 图 $6 \mathrm{~b}$ 中介质群的运动 区域分布特征与图 6a 类似。不同的是下落的介质群 对物料的破碎方式是冲击破碎, 而水平方向运动的 介质群破碎物料的方式则是剪切研磨 [30]。依据离散 介质群动力学 [31], 介质群之间有力作用的相互接触 才能对物料起到冲击或研磨的破碎作用, 即介质群

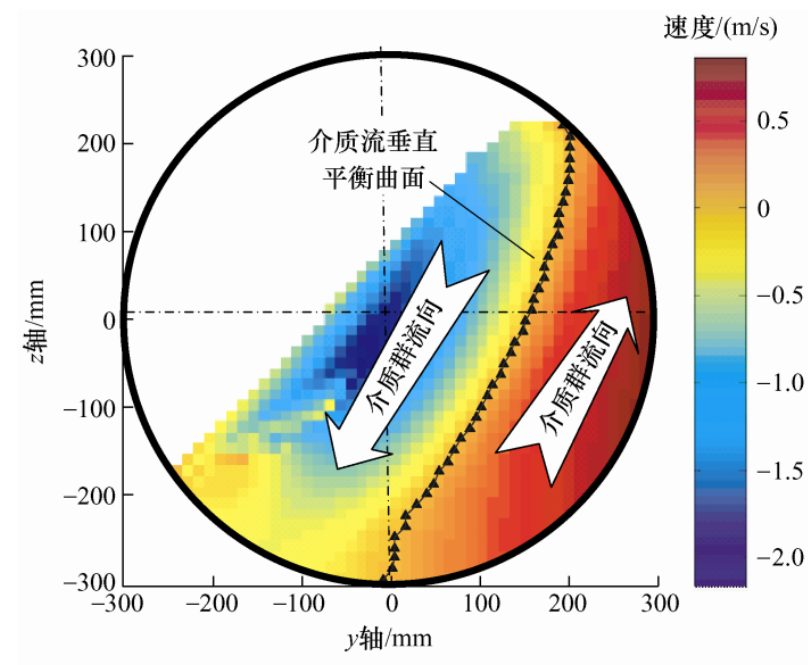

(a) 介质群垂直方向 $(z$ 轴)速度
速度变化梯度是介质群之间相互作用力的体现。如 图 6c 中介质群平衡面上方区域的介质群速度梯度 变化较大, 第一象限中介质群速度由小变大, 是由 于重力做功使动能增加, 而第三象限中速度由大减 小, 说明该区域的动能转化为碰撞能, 可能是物料 破碎的有效区域。因此需要对介质群的碰撞能量作 进一步分析。

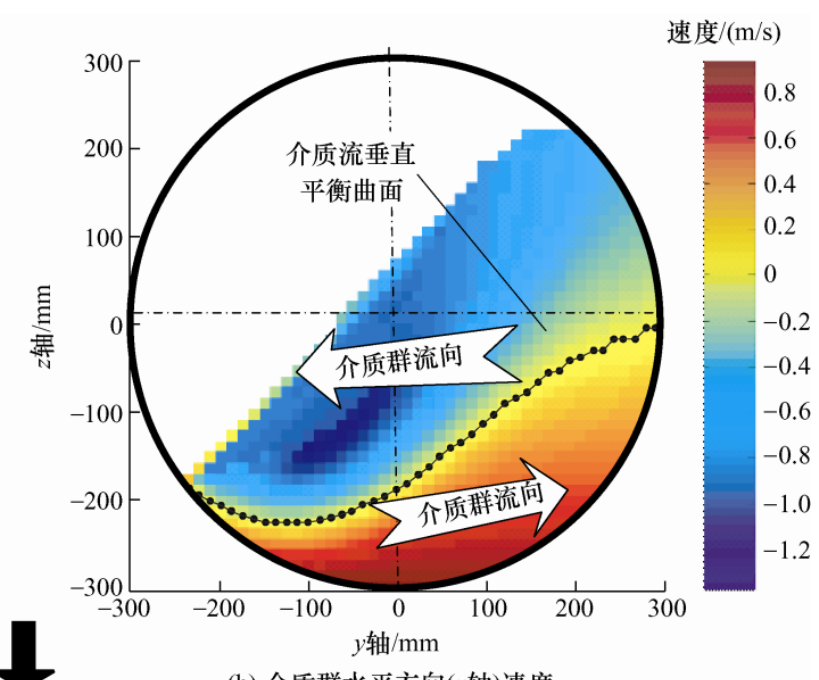

(b) 介质群水平方向 $(y$ 轴)速度

速度 $/(\mathrm{m} / \mathrm{s})$

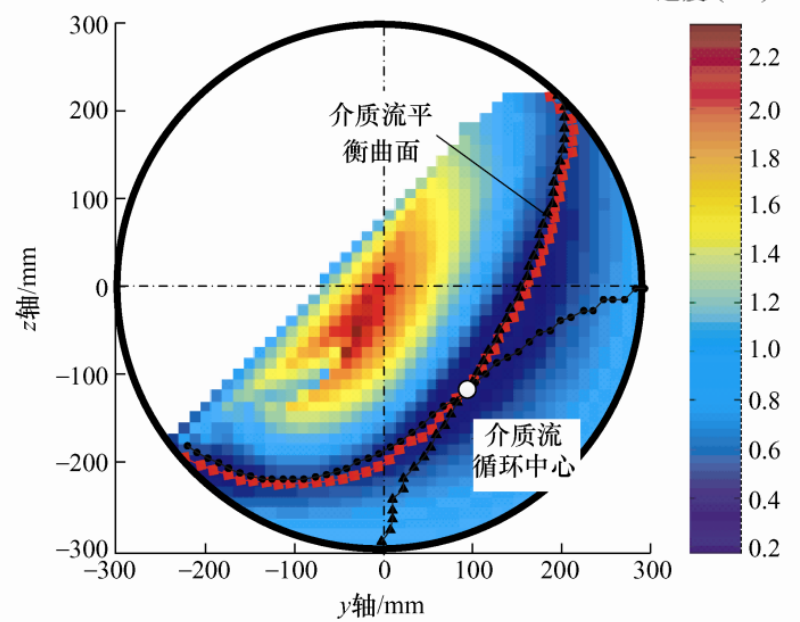

(c) 介质群总速度

图 6 一个稳定运动周期中回转运动中介质群运动速度矢量区域分布

\section{2 碰撞特性区域分布}

根据牛顿第二定律, 介质群的运动速度直接决 定着介质间的相互作用力, 速度分布特征也间接表 征着介质群的碰撞分布特性。球磨过程中介质间的 碰撞种类主要有法向碰撞和切向碰撞两种, 且不同 区域的法向和切向碰撞频次不同，对物料的破碎作 用也有明显差异。过大的碰撞能会导致颗粒过粉碎, 而过小的碰撞能无法有效破碎物料 [31]。因此, 适当 大小的碰撞能级才能产生并积累特定粒径的粉体颗
粒。这里把 “适当大小” 的碰撞能级定义为最佳碰 撞能，最佳碰撞能级分布的区域定义为活性区域。 下文粉磨试验中选取建筑砂为粉磨进料, 其最佳碰 撞能量范围为 $0.01 \sim 0.10 \mathrm{~J}^{[29]}$ 。

图 7 中网格的位置和颜色等级分别代表该参数 的分布区域和能级大小。图 7a 7d 分别是介质群法 向碰撞能级、切向碰撞能级、碰撞频次以及最佳碰 撞能级的区域分布图, 图中各碰撞能级的区域范围、 位置及能量密集区域都可通过模型图直观表达。由 


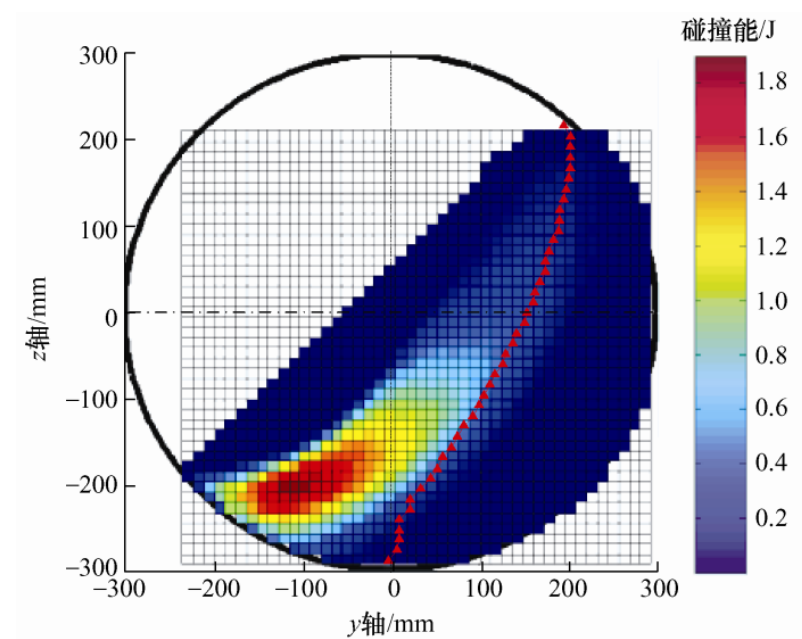

(a) 法向碰撞能

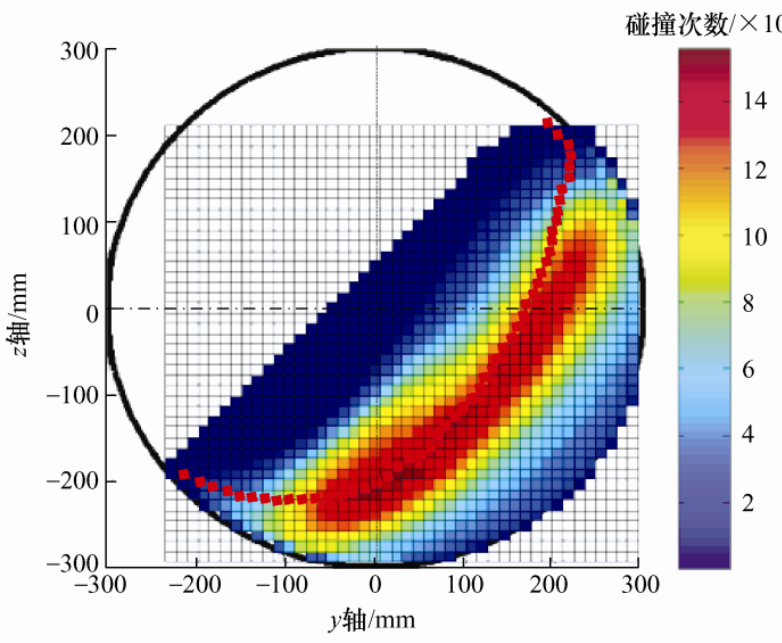

(c) 碰撞频次

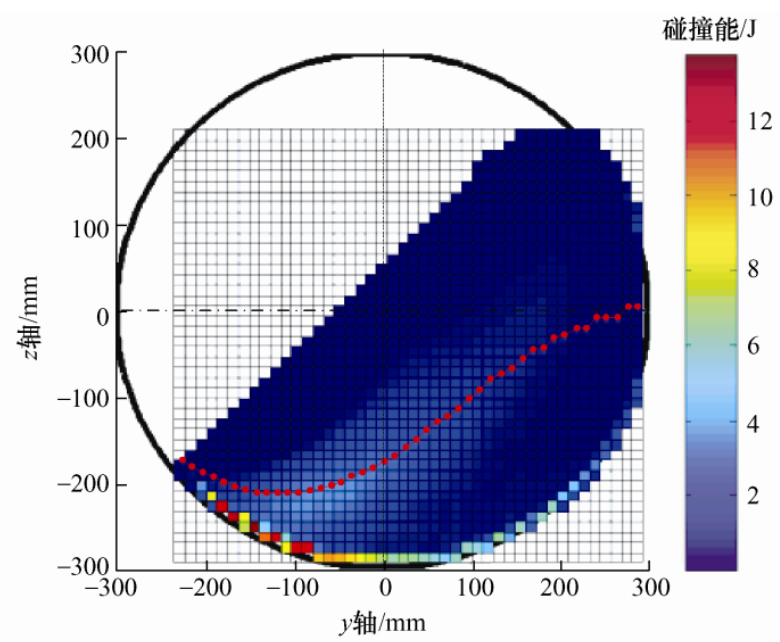

(b) 切向碰撞能

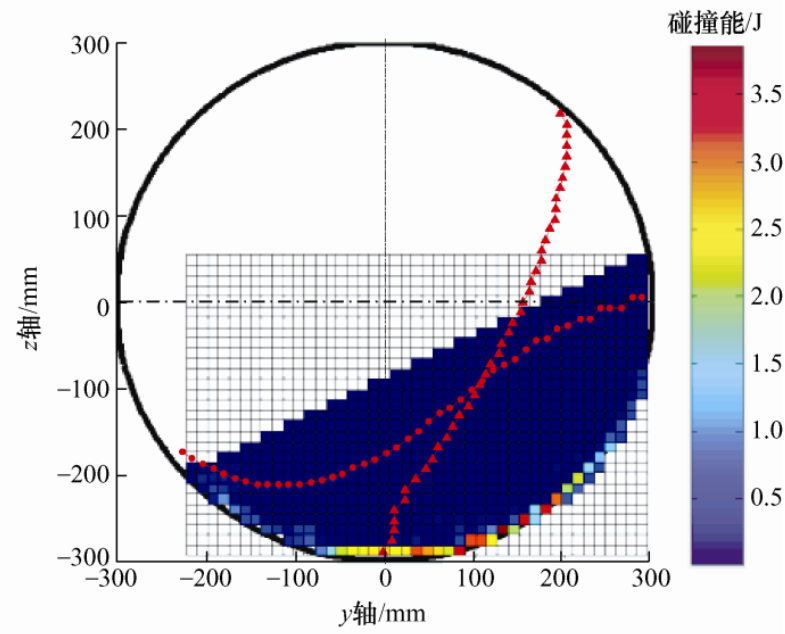

(d) 最佳碰撞能

图 7 一个稳定运动周期中回转运动中介质群的碰撞特性区域分布

图 6 的分析结果可知: 碰撞能级的区域分布与介 质群的运动速度梯度变化和碰撞频次密不可分, 图 7a $7 \mathrm{~b}$ 显示法向碰撞能和切向碰撞能的密集 区域主要分布在第三象限的介质群运动平衡面 附近, 而图 7c 显示介质群运动平衡面是碰撞频 次最高的区域。由此说明速度梯度变化、碰撞频 次与介质群的碰撞能级呈正相关。同时, 图 $7 \mathrm{~d}$ 是根据建筑砂的最佳碰撞能级范围得到的能够 使其有效破碎的活性区域分布图。

\section{3 介质运动区域划分实例及粉磨试验}

\section{1 不同工况下介质运动区域划分实例}

为验证 EDEM 网格划分法能够适应不同工况 的球磨过程, 选取三种不同运动模式: 单一回转运 动、螺旋运动(角螺旋祄板 ${ }^{[14]}$ ) 和复合颤振运动 ${ }^{[30]}$, 上文已对回转运动中介质群运动区域进行了划分, 这里给出另外两种运动模式中介质群的区域分布情
况, 图 8〜9 是介质群速度矢量区域分布图, 图 10〜 11 是介质群碰撞特性区域分布图。

图 8９ 中介质群运动速度区域分布差异性显 著, 以运动平衡面为界, 图 8 中两个不同运动方向 的区域范围差异较大, 而图 9 中的两个区域范围更 趋向均衡。从图 $8 \mathrm{a} \sim 8 \mathrm{~b}$ 中灰度等级变化可以看出介 质群平衡面下方区域的速度变化梯度较大, 即介质 群的剪切研磨作用较强, 但分布范围相对较小。而 平衡面上方的速度梯度变化较小, 但区域分布大, 即冲击作用弱, 作用范围大。图 8c 中介质群平衡面 上方区域的速度梯度变化较大, 且集中分布在平衡 面末端。结合图 10 中介质群的碰撞能级分布特性: 法向碰撞能分布在平衡面末端, 切向碰撞能更靠近 筒体底部区域, 最佳碰撞能的分布区域也较大, 说 明此工况下介质群冲击和研磨破碎作用都较强, 且 作用区域大, 可能对物料的破碎作用较好。图 9 中 速度梯度分布特征是: 介质流平衡面上方的速度梯 度变化较大, 且在第三象限的位置速度梯度变化最 
集中。结合图 11 中的碰撞能量分布特性: 法向碰撞 能级较小, 切向碰撞能级较大, 但区域分布都较小,
说明此工况下介质群的冲击作用弱, 剪切研磨作用 较强, 且破碎区域明显减小, 局部分布在筒壁附近。

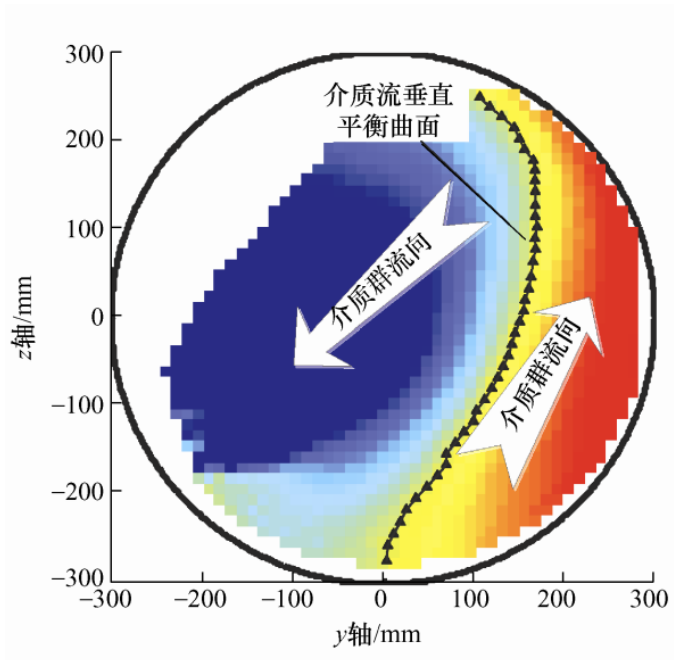

(a) 介质群垂直方向( $z$ 轴)速度

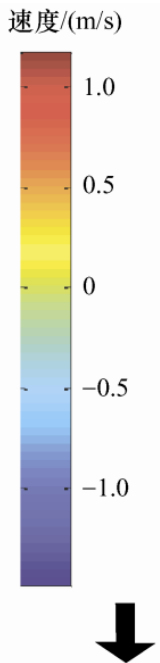

4

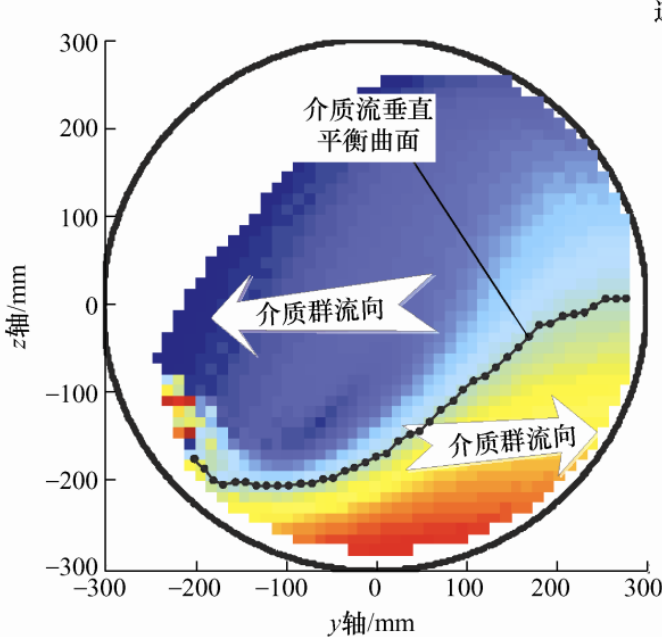

(b) 介质群水平方向 $(y$ 轴)速度
速度 $/(\mathrm{m} / \mathrm{s})$

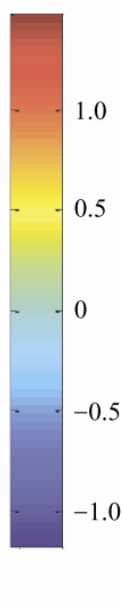

速度 $/(\mathrm{m} / \mathrm{s})$

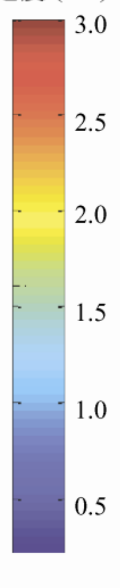

(c) 介质群总速度

图 8 螺旋运动中介质群运动区域分布图(以四边形角螺旋祄板为例)

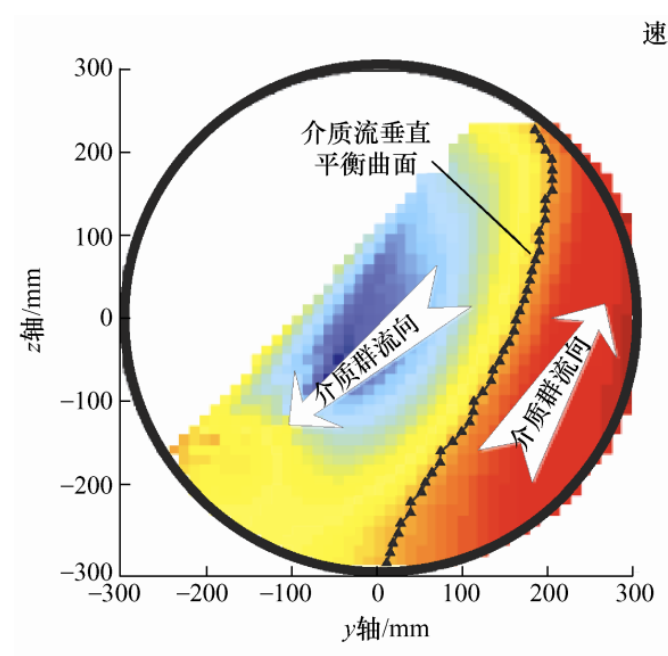

(a) 介质群垂直方向 $(z$ 轴)速度

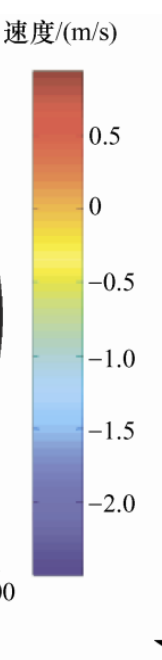

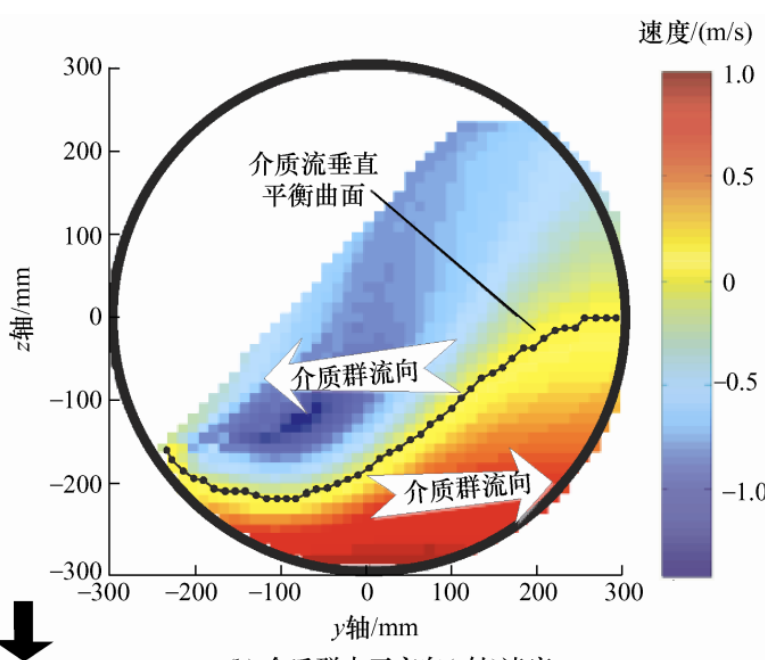

(b) 介质群水平方向 $(y$ 轴)速度 


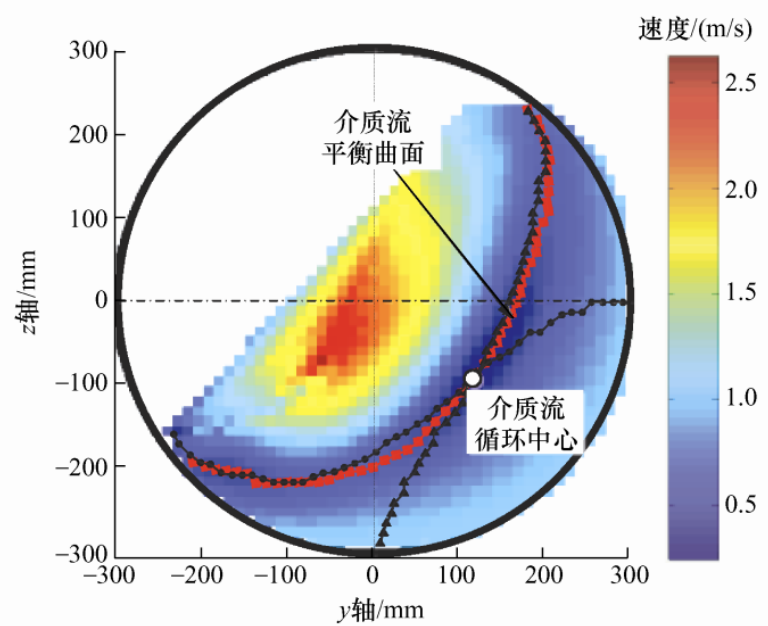

(c) 介质群总速度

图 9 复合颤振运动中介质群运动区域分布图(以颤振球磨机为例)

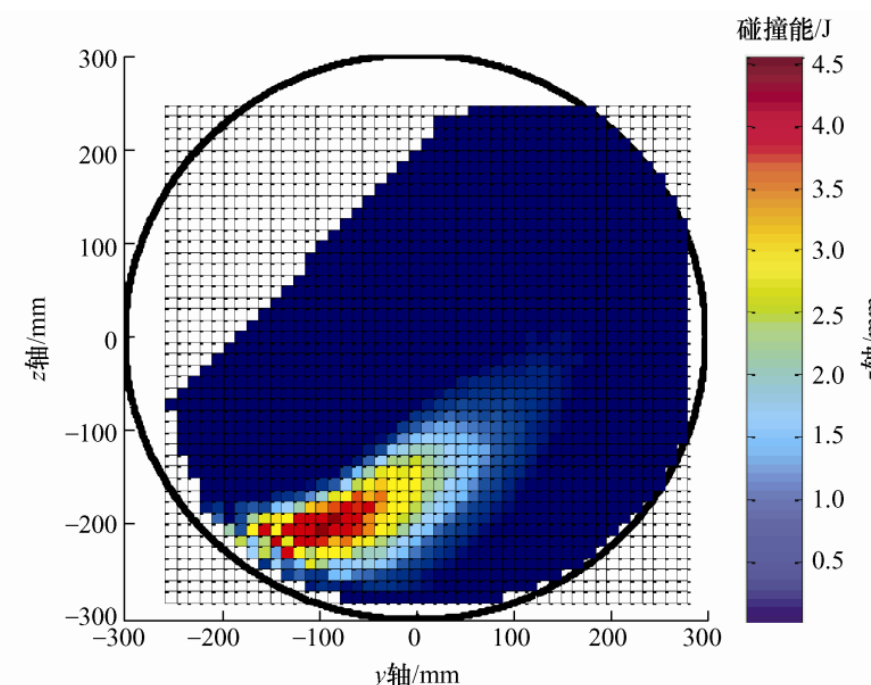

(a) 法向碰撞能

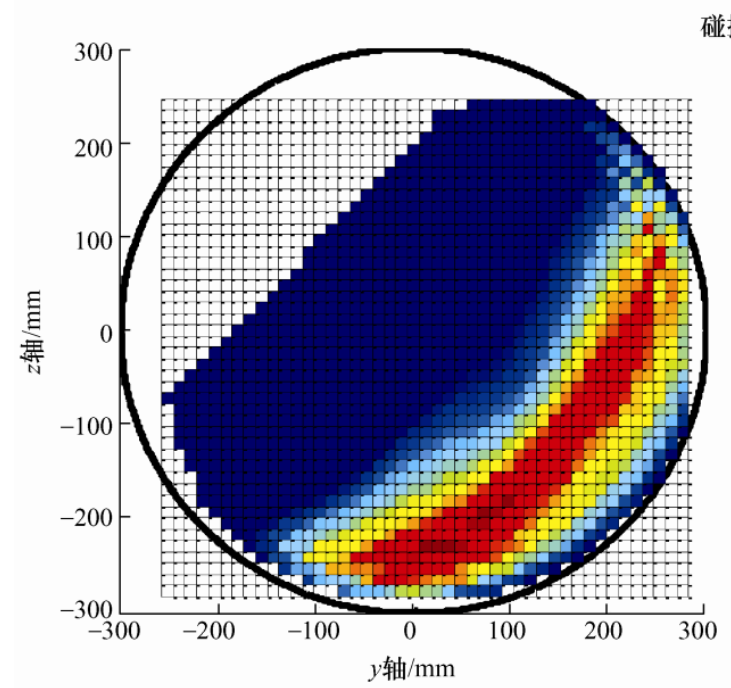

(c) 碰撞频次
碰撞次数 $/ \times 10^{4}$
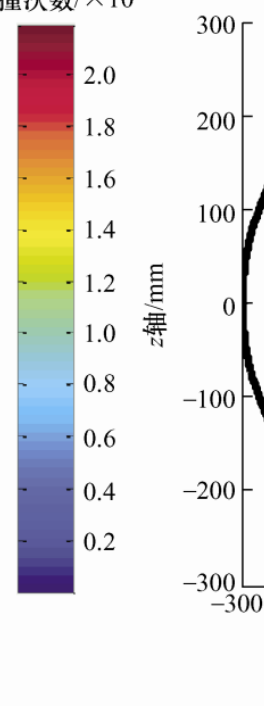

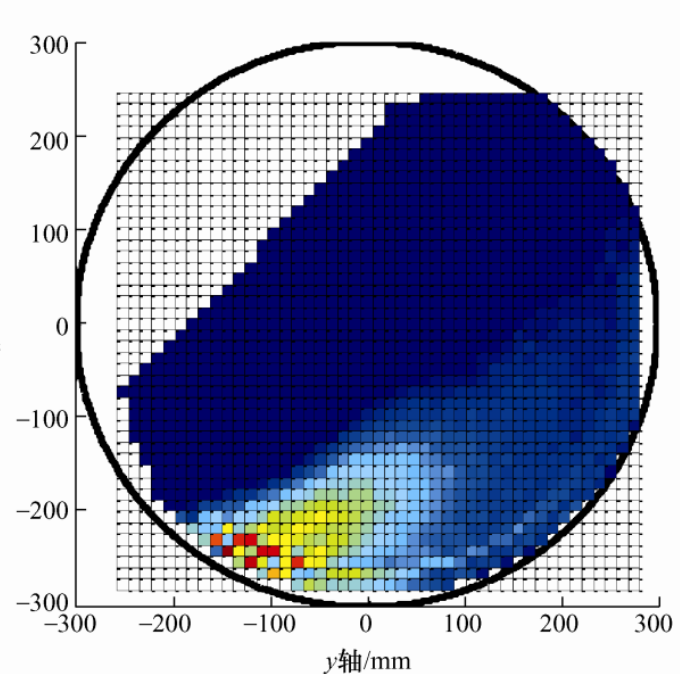

(b) 切向碰撞能

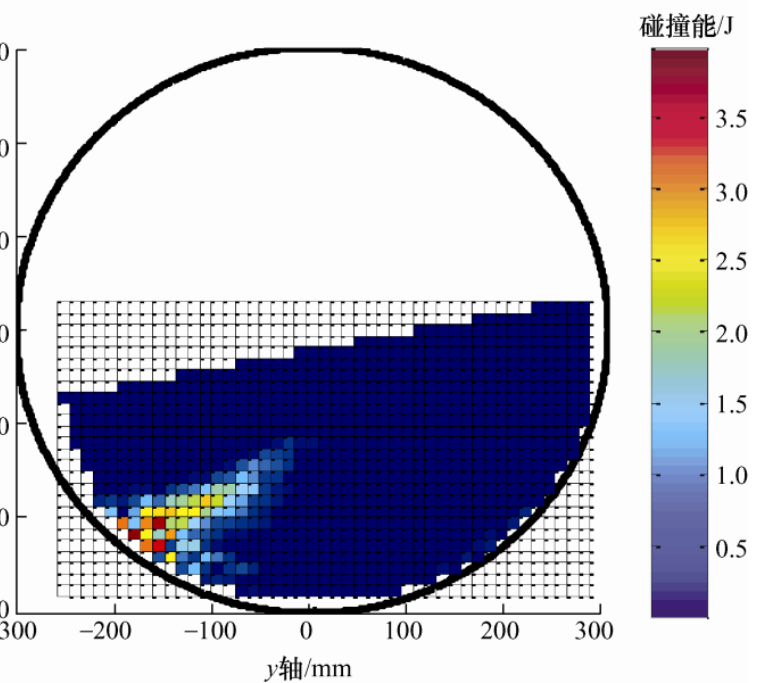

(d) 最佳碰撞能

碰撞能/J

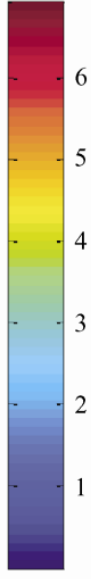

碰撞能/J

\section{0}

\section{5}

0

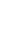

(1)




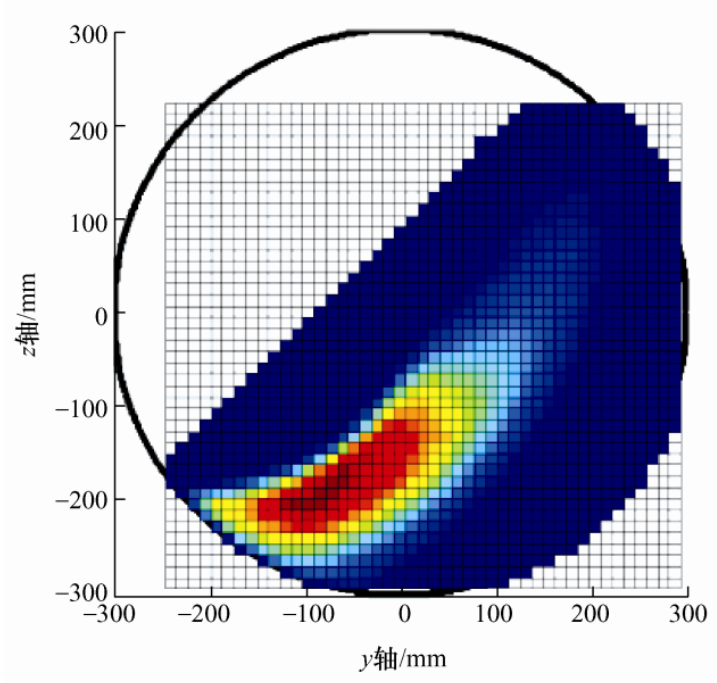

(a) 法向碰撞能

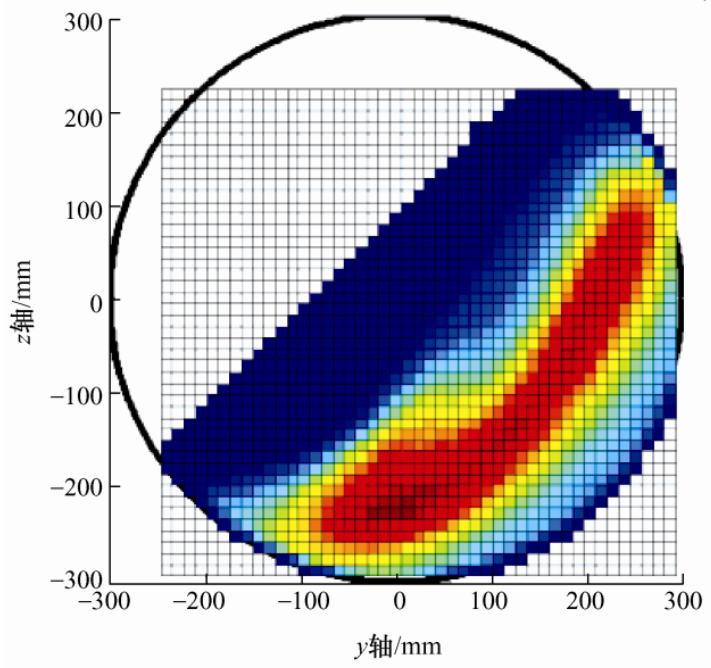

(c) 碰撞频次

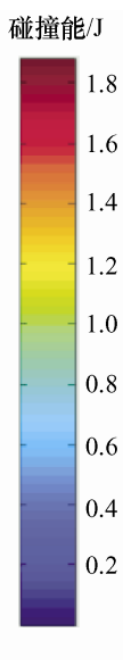

碰撞次数 $/ \times 10^{4}$

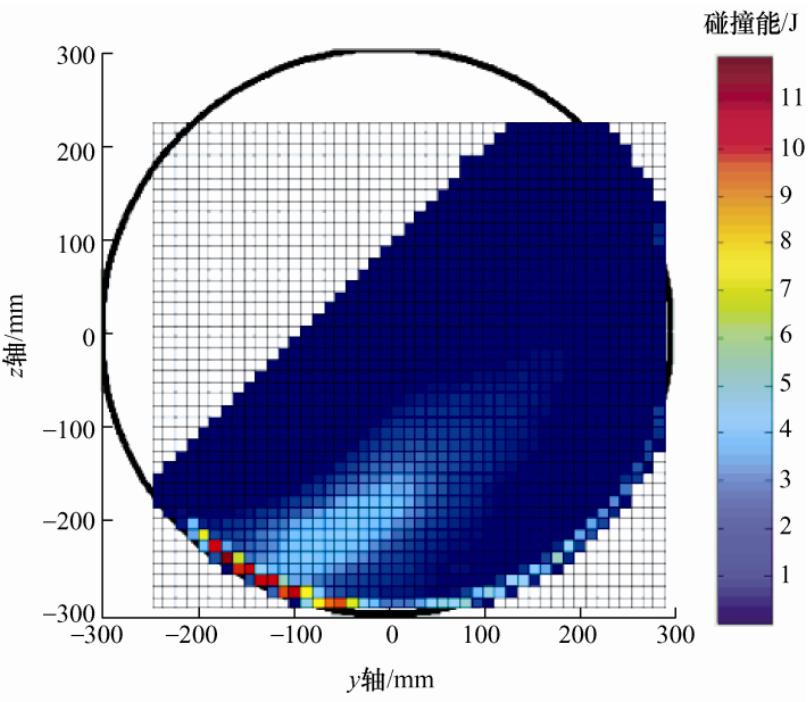

(b) 切向碰撞能

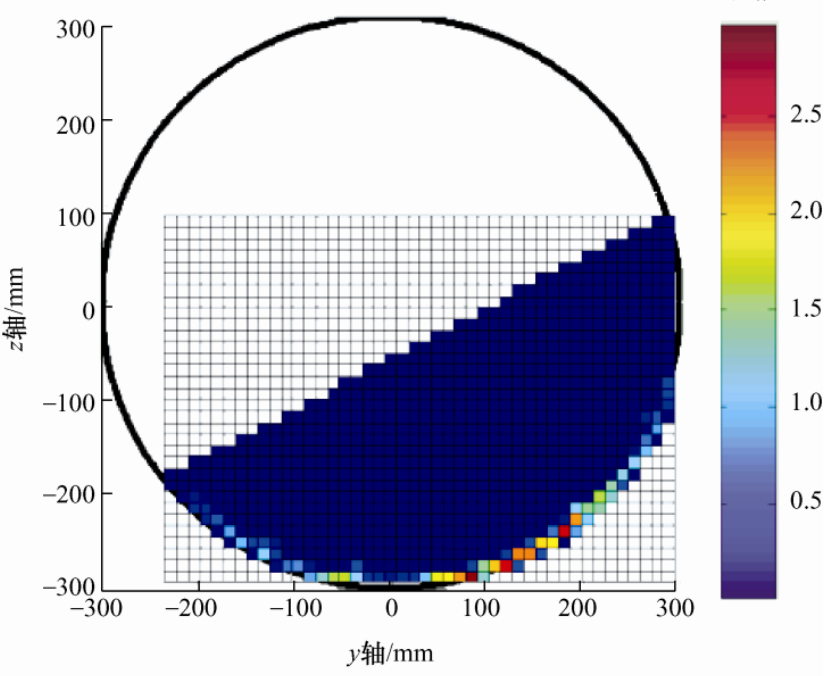

(d) 最佳碰撞能

图 11 复合颤振运动中介质群碰撞特性区域分布

比较回转运动(图 6 和图 7)、螺旋运动(图 8 和 图 10)以及复合颤振运动(图 9 和图 11)中的介质群运 动区域特性, 可知螺旋运动中介质群运动区域最大, 介质流垂直平衡曲面上方和水平平衡曲面下方区域 的速度变化梯度(由大变小)最明显, 对应的法向和 切向碰撞能级及分布区域均较大; 复合颤振运动中, 介质群的上述运动特性相对较弱, 而回转运动中最 不明显。

\section{2 粉磨试验}

\subsection{1 试验装置及操作参数}

试验球磨机如图 12 所示, 该装置分别通过转 动电动机及转动电动机与振动电动机的耦合实现 筒体的回转运动和复合颤振运动。试验中设置 $X$ 、 $Y 、 Z$ 三个方向的加速度传感器用来检测筒体的振 动幅度, 监测点 $A 、 B 、 C$ 的固定位置和控制系统 部分见图 12 中细节放大图所示。试验进料建筑砂
的主要矿物成分是 $\mathrm{SiO}_{2}$ 。球磨过程中试验磨机的 操作条件和试验参数见表 1 。

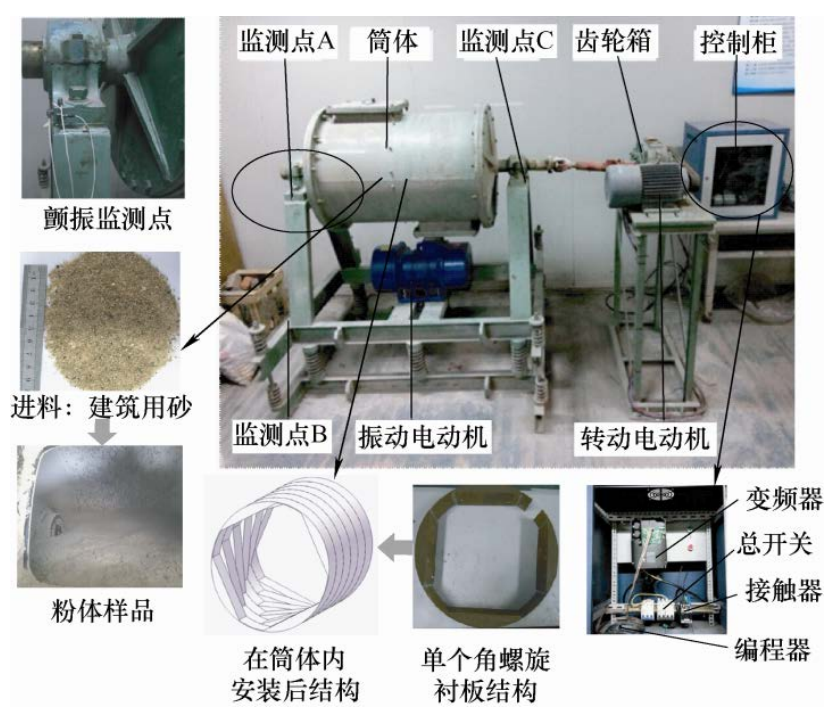

图 12 试验球磨机及系统装置图 
表 1 设备操作条件和试验参数

\begin{tabular}{cc}
\hline 参数名称 & 数值 \\
\hline 磨筒直径 $\Phi / \mathrm{mm}$ & 600 \\
填充率 $(\%)$ & 45 \\
介质尺寸 $/ \mathrm{mm}$ & $\phi 20: \phi 16: \phi 10$ \\
介质配比 & $3: 6: 1$ \\
介质材质 & 合金钢 \\
密度 $/\left(\mathrm{kg} / \mathrm{m}^{3}\right)$ & $7.8 \times 10^{3}$ \\
进料 & 建筑用砂 $\left(\mathrm{SiO}_{2}\right)$ \\
粒径范围 $/ \mathrm{mm}$ & $0.4 \sim 0.6$ \\
\hline
\end{tabular}

\subsection{2 试验方案及粉体样品测量}

针对上述不同工况下介质群运动区域分布的差 异性, 设计对应的筒体运动方案: 单个转动电机实 现介质群的纯回转运动; 转动电机结合角螺旋祄板 实现介质群的螺旋回转运动; 转动电机耦合振动电 机实现介质群的复合颤振运动。具体试验点设计如 表 2 所示。试验过程中按设定取样时刻, 每隔 $5 \mathrm{~min}$ 取样一次, 每次取样 $50 \mathrm{~g}$, 粉磨总时间 $85 \mathrm{~min}$ 。对 样品冷却、干燥处理后进行颗粒粒径分析、比表面 积测量和电镜扫描(SEM)图像拍摄。样品的各项测 试仪器名称及型号见表 3 。

表 2 试验方案设计

\begin{tabular}{ccccc}
\hline 试验点 & 介质群运动 & 转速率 $(\%)$ & 振幅 $/ \mathrm{mm}$ & 频率 $/ \mathrm{Hz}$ \\
\hline 1 & 回转运动 & 85 & - & - \\
2 & 螺旋运动 & 85 & - & - \\
3 & 复合㗜振 & 85 & $4 \sim 6$ & $8 \sim 12$ \\
\hline
\end{tabular}

表 3 粉体样品测试仪器

\begin{tabular}{cc}
\hline 名称 & 型号 \\
\hline 振动测试仪 & Triaxial ICP ${ }^{\circledR}$ Accelerometers \\
马尔文激光粒度分析仪 & Scirocco2000(A) \\
比表面积分析仪 & ASAP2020 \\
台式铇灯丝扫描电镜 $(\mathrm{SEM})$ & VEGA3 SBH \\
\hline
\end{tabular}

\section{4 试验结果分析与讨论}

\section{1 试验结果分析}

\subsection{1 破碎颗粒粒径分布}

图 13 是三种运动形式下破碎颗粒的粒径分 布图。图中曲线分布形态显示三种工况中粉磨的 微细颗粒的粒径均服从 RRSB 分布 ${ }^{[31]}$ 。区别在于: 当粒径 $d<4 \mu \mathrm{m}$ 时, 螺旋运动中的微细颗粒体积 累积百分比明显高于其他两种运动, 粒径 $d>$ $4 \mu \mathrm{m}$ 时, 复合颤振运动中的微细颗粒体积累积百 分比趋于与螺旋运动重合, 而回转运动的体积累
积百分比曲线增长缓慢, 说明回转运动中的粗粒 径颗粒占比大。

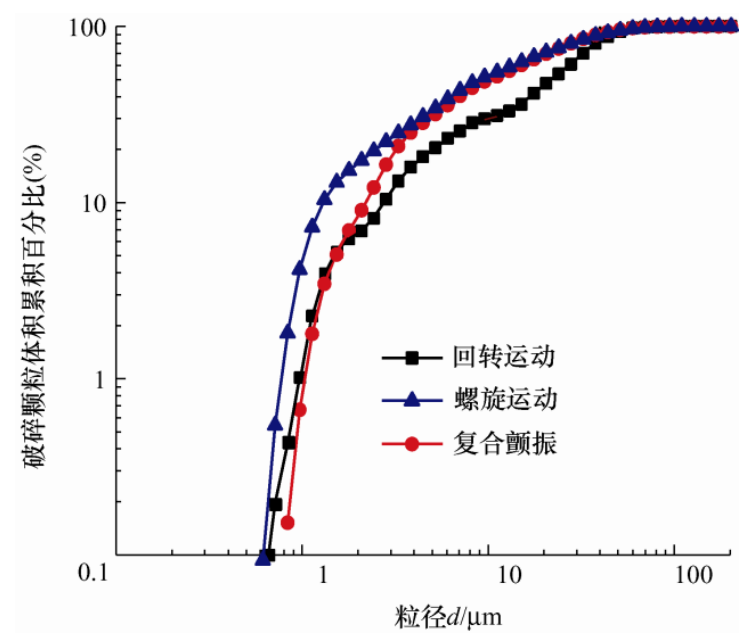

图 13 破碎颗粒粒径分布曲线

\subsection{2 破碎率}

基于戴维斯的一级粉磨动力学方程 ${ }^{[32]}$, 当进料 的粒径范围很小时, 可近似看作是同一粒径的颗粒, 此时 $i=1$, 戴维斯动力学方程可表示为

$$
\ln \frac{M_{1}(t)}{M_{1}(0)}=\frac{-S_{1} t}{2.3}
$$

式中, $M_{1}(0)$ 和 $M_{1}(t)$ 表示进料颗粒粉磨前和粉磨 $t$ 分钟后的质量百分比。对各组试验中获取的数据点 进行线性拟合, 得到进料粒径的一级粉磨动力学曲 线图, 如图 14 所示。拟合系数 $R^{2}$ 均近似等于 1 。 拟合曲线的斜率即为式(4)中系数 $S_{1}$, 也即是进料颗 粒(粗颗粒)减少的速率，称之为破碎率。三种运动 模式下进料的破碎率 $S_{1}$ 如图 15 所示, 螺旋运动中 的破碎率最大, 复合颤振运动次之, 回转运动中进 料的破碎率最小。

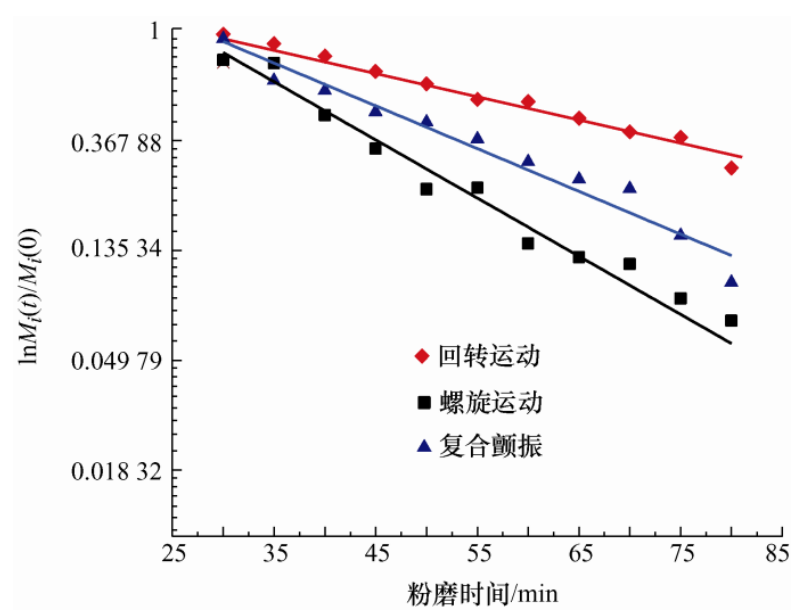

图 14 进料粒径的一级粉磨动力学曲线 


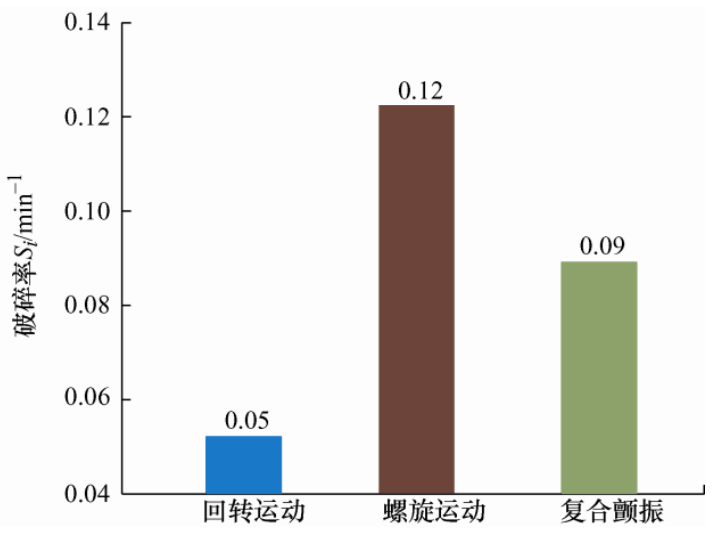

图 15 进料颗粒的破碎速率 $S_{1}$

\subsection{3 破碎颗粒微观形态}

图 16 给出了三种运动模式中破碎颗粒的 SEM 图像。图像中微细颗粒的粒径大小以及粒径分布均 匀度较好的工况均是在螺旋运动中, 复合颤振中微 细颗粒的均匀度较好, 但是粒径较大。虽然回转运 动中粒径较细, 但粒径分布不均匀, 细颗粒过细,
而粗颗粒又较粗，粒径分布的范围大。不同运动模 式下破碎颗粒的微观形态图与图 13 中颗粒粒径分 布曲线图基本吻合。

\subsection{4 微细颗粒产率}

图 17 给出了三种运动模式中微细颗粒的产率, 即随着粉磨时间的增加，325目 $(45 \mu \mathrm{m}) 、 650$ 目 (21 $\mu \mathrm{m})$ 以及 1600 目 $(10 \mu \mathrm{m})$ 颗粒的累积体积/质量 百分比。同样地，根据各组试验中获取的数据，拟 合数据点得到不同工况下的产率曲线, 如图 17a 17c 所示。不同粒径产品的产率拟合方程及拟合系 数参见表 4。由于图 17a 中线性拟合方程的拟合系 数 $R^{2} \approx 0.83$, 相比较其他情况, 其拟合系数较低, 说 明线性拟合不合理。因此，采用 Sigmoid 函数(S 型 生长曲线)对图 17a 的数据点进行重新拟合, 得到的 拟合曲线图如图 $17 \mathrm{~d}$ 所示，拟合系数 $R^{2} \approx 1$ 。

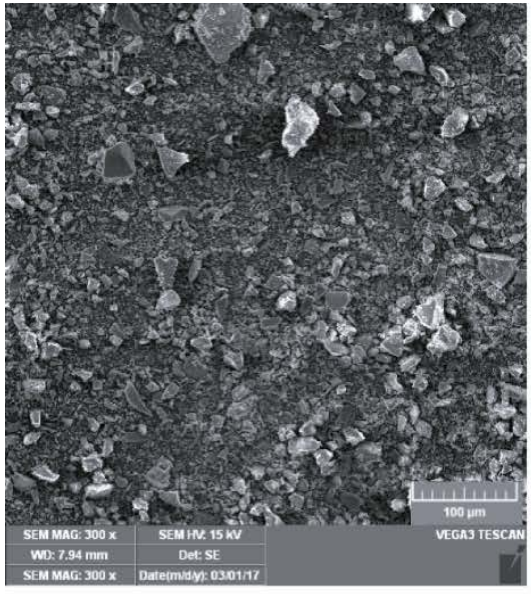

(a) 回转运动

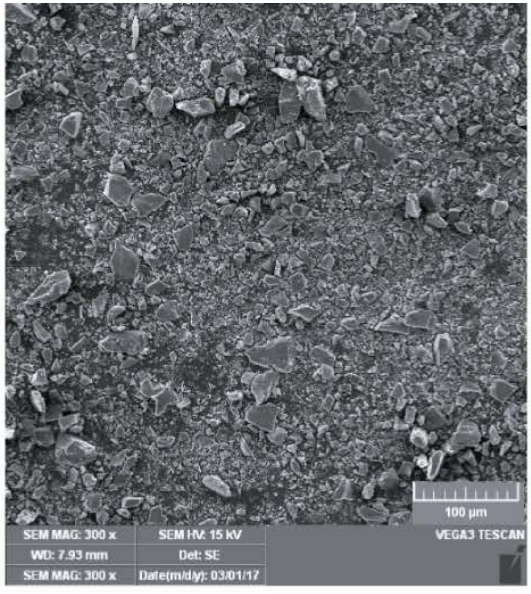

(b) 螺旋运动

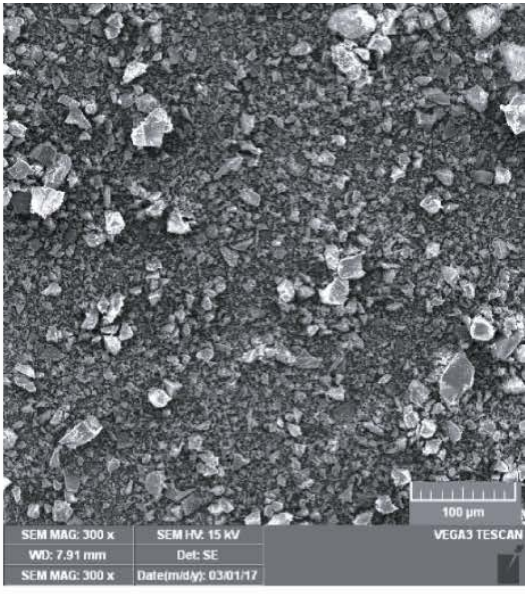

(c) 复合颤振运动

图 16 破碎颗粒的 SEM 图

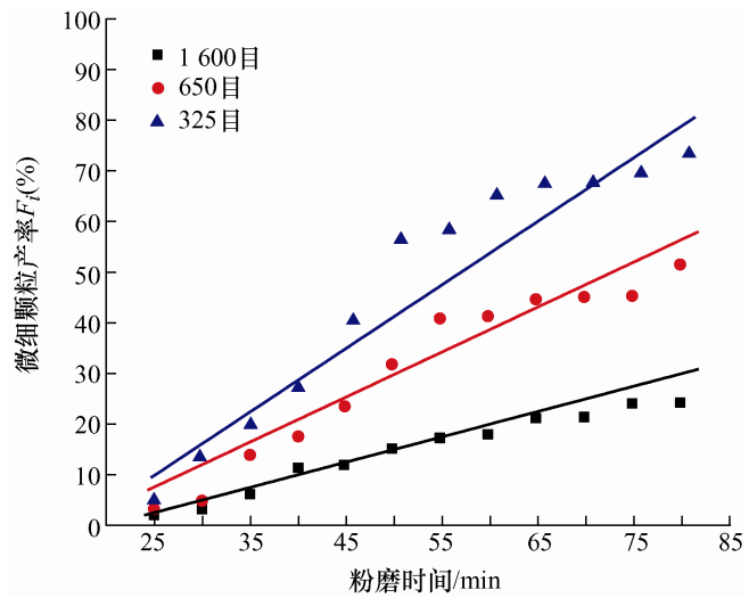

(a) 回转运动

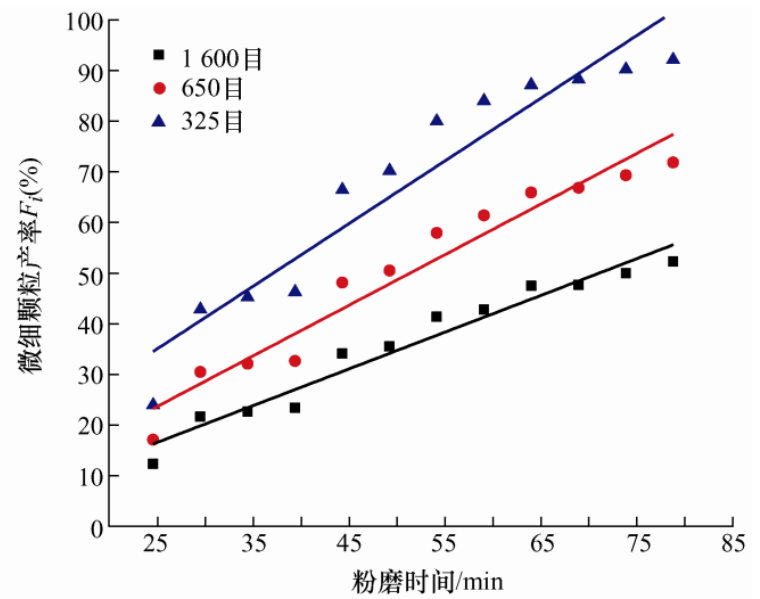

(b) 螺旋运动 


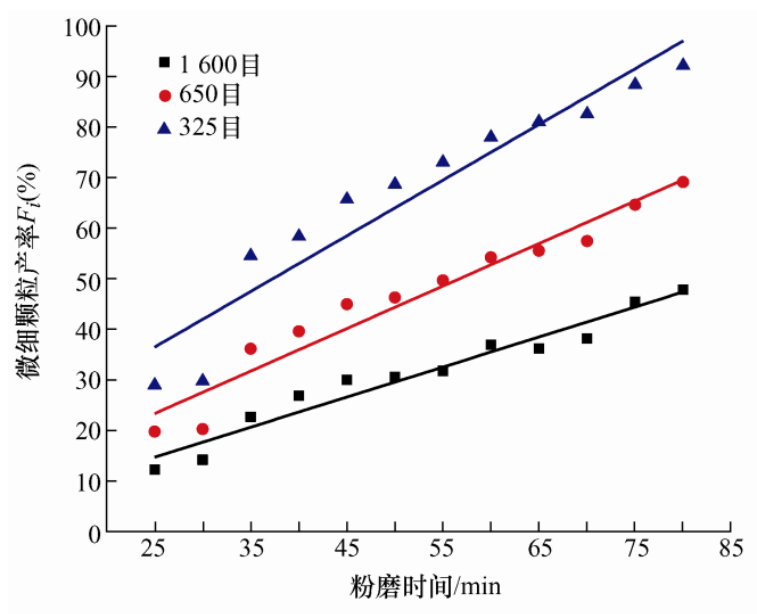

(c) 复合颤振运动

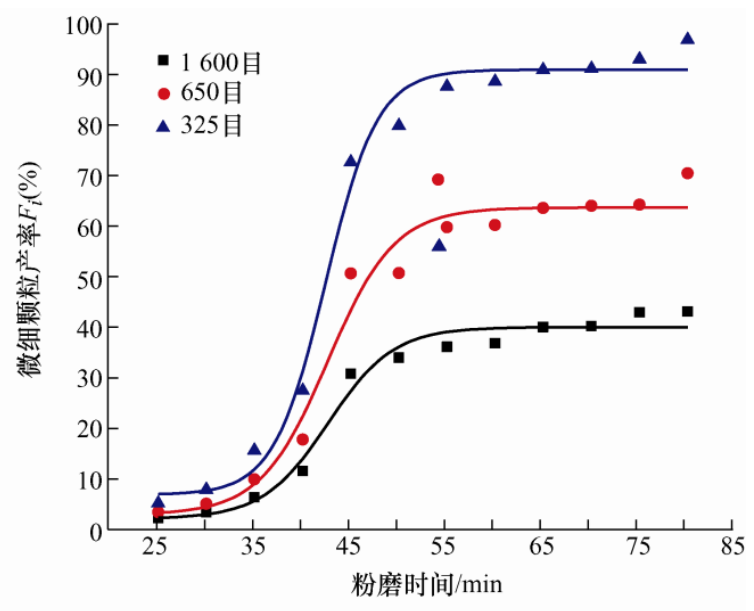

(d) 对17a中数据点进行曲线拟合结果

图 17 三种运动模式下三种粒径的微细颗粒的产率变化情况

表 4 微细颗粒产率的拟合曲线及拟合系数

\begin{tabular}{ccccc}
\hline \multirow{2}{*}{ 试验点 } & \multicolumn{3}{c}{ 不同粒径产率的拟合方程 } & 拟合系数 \\
\cline { 2 - 4 } & 325 目 & 650 目 & 1600 目 & $R^{2}$ \\
\hline 1 & $y=0.7 x-26$ & $y=0.5 x-17$ & $y=0.3 x-8.9$ & 0.83 \\
2 & $y=1.2 x+2.7$ & $y=x-2.8$ & $y=0.7 x-3.0$ & 0.92 \\
3 & $y=1.1 x+9.9$ & $y=0.8 x+3.3$ & $y=0.6 x+0.8$ & 0.93 \\
\hline
\end{tabular}

\section{2 试验结果讨论}

通过上述不同运动模式下对进料颗粒的破碎效 果及破碎速率的试验分析, 结合 3.1 和 3.2 节的介质 群运动区域划分以及碰撞活性区域的分布特征, 可 知: 介质群运动区域分布范围最大的螺旋运动中, 由于介质群的冲击、研磨破碎作用都较强, 物料的 破碎速率和微细颗粒的产率都最大, 说明介质群运 动平衡面的位置及速度梯度的变化决定着碰撞能级 和区域分布, 更是分析物料有效破碎的关键因素。

回转运动中, 介质群的运动区域分布最小, 介 质流垂直平衡面上方的冲击区域(法向碰撞)及水平 平衡面下方的研磨区域均较小, 但水平平衡面下方 的速度梯度变化较均匀, 连续变化的速度使切向碰 撞能级分布均匀, 对各种粒径的颗粒都有较好的研 磨作用或二次研磨破碎, 恰好验证了粉磨试验的结 果(图 17): 在粉磨初始阶段(前 $40 \mathrm{~min}$ ), 回转运动 中主要是冲击作用导致的粗颗粒破碎, 微细颗粒累 积很少, 粉磨一段时间后, 由于研磨或二次破碎作 用, 使更多细粒径颗粒产生与累积, 产率迅速增加, 如图 17d 所示。但是由于作用区域小, 对颗粒的冲 击、研磨破碎区域均有限, 导致细颗粒被过度破碎, 粒径过细, 粗颗粒没有被充分破碎的现象, 所以回转 运动中三种微细颗粒的产率差异最明显 SEM 图像中也 可以明显看出粒径大小的差异性。同时, 由于活性区 域(最佳碰撞能)最小, 产率是三中工况中最低的。
复合颤振运动中介质流垂直平衡面上方的速度 梯度变化较大(法向碰撞), 但分布区域较小, 因此 在粉磨初始阶段(前 25 30 min)冲击破碎产生的细 颗粒较少，但由于水平平衡面下方的研磨区域及速 度变化梯度相对回转运动中都有所增加, 颗粒的研 磨破碎或二次破碎概率上升, 慢慢产生并积累更多 的微细颗粒, 致使产率增加, 如图 17c 所示。

综上所述，介质群的速度区域分布特性及介质 流平衡面分布对介质群的碰撞能级、区域分布及活 性区域均具有重要影响, 继而直接影响着颗粒的破 碎效果及破碎速率。

\section{5 结论}

(1) 通过 EDEM 网格划分法得到的介质群运动 区域分布, 有效地反映了实际工况中的介质群的运 动状态，且操作简单、适用性强。

（2）介质群的速度区域分布、速度梯度及介质 流平衡面直接影响着介质群的碰撞能级、区域分布 及活性区域，继而决定着介质群对物料的破碎效果 和破碎速率。

(3) 通过 EDEM 网格划分法获取的介质群运动 速度矢量分布区域和碰撞特性分布区域可以直观、 有效的显示介质群对物料的破碎方式和破碎区域, 为开展不同工况中球磨过程的破碎机理研究提供了 简便、可行的技术指导。

\section{参 考 文 献}

[1] 盖国胜, 陶珍东. 粉体工程[M]. 北京：清华大学出版 社, 2009.

GAI Guosheng, TAO Zhendong. Powder technology[M]. Beijing: Tsinghua University Press, 2009. 
[2] 罗春梅, 肖庆飞, 段希祥, 等. 球磨机功能转变与节能 途径分析[J]. 矿山机械, 2011，39(1): 81-84.

LUO Chunmei, XIAO Qingfei, DUAN Xixiang, et al. Analysis of function transformation and energy saving ways of ball mill[J]. Mining \& Processing Equipment, 2011, 39(1): 81-84.

[3] 夏恩品, 董为民. 影响球磨机介质运动规律主要因素的 试验研究[J]. 矿山机械, 2009, 37(19): 60-63.

XIA Enpin, DONG Weimin. Experimental research of the main factors in influencing the medium movements in the ball mill[J]. Mining \& Processing Equipment, 2009, 37(19): 60-63.

[4] TORAMAN O Y, KATIRCIOGLU D. A study on the effect of process parameters in stirred ball mill[J]. Advanced Powder Technology, 2011，22(1): 26-30.

[5] 卢建坤. 基于离散单元法的大型球磨机介质运动分析 及参数优化 $[D]$. 洛阳: 河南科技大学, 2013 .

LU Jiankun. Kinematical analysis \& parameters optimization of large tumbling ball mill' media based on the discrete element method[D]. Luoyang: Henan University of Science and Technology, 2013.

[6] 李永堂, 付建华, 雷步芳, 等. 多元低合金耐磨钢破碎机 祄板制造工艺研究[J]. 机械工程学报, 2013, 49(12): 72-77. LI Yongtang, FU Jianhua, LEI Bufang, et al. Research on manufacturing process for complex low-alloyed wear-resisting crusher liner[J]. Journal of Mechanical Engineering, 2013, 49(12): 72-77.

[7] OWEN P, CLEARY P W. The relationship between charge shape characteristics and fill level and lifter height for a SAG mill[J]. Minerals Engineering, 2015， 83(11): 19-32.

[8] GUPTA V K, SHARMA S. Analysis of ball mill grinding operation using mill power specific kinetic parameters[J]. Advanced Powder Technology, 2014， 25(2): 625-634.

[9] SABAH E, ÖZDEMIR O, KOLTKA S. Effect of ball mill grinding parameters of hydrated lime fine grinding on consumed energy[J]. Advanced Powder Technology, 2013, 24(3): 647-652.

[10] KIANGI K K, MOYS M H. Particle filling and size effects on the ball load behavior and power in a dry pilot mill: Experimental study[J]. Powder Technology, 2008, 197(1): 79-87.

[11] 刘军德. 基于 MEMS 的球磨机介质运动测量技术研究 [D]. 杭州: 浙江工业大学, 2012.

LIU Junde. The research of ball mill medium motion measurement based on MEMS[D]. Hangzhou: Zhejiang
University of Technology, 2012.

[12] LI N, SUN Y, et al. A finite element analysis on effects of alternating load on cylinder of a vibrating ball milling[J]. Procedia Engineering, 2011, 16(1): 95-100.

[13] POWELL M S, WEERASEKARA N S, COLE S, et al. DEM modelling of liner evolution and its influence on grinding rate in ball mills[J]. Minerals Engineering, 2011, 24(3-4): 341-351.

[14] 梁曼, 孙毅, 纪朋朋, 等. 正多边形角螺旋祄板对球磨 机粉磨效率影响的数值分析 [J]. 机械工程学报, 2015, 51(17): 203-212.

LIANG Man, SUN Yi, JI Pengpeng, et al. Numerical analysis on regular polygon angle-spiral liners design in a ball mill[J]. Journal of Mechanical Engineering, 2015, 51(17): 203-212.

[15] 樊狄锋, 孙毅, 毛亚郎, 等. 球磨机混合运动状态下介质 运动形态的分析研究[J]. 矿山机械, 2010, 38(5): 81-83. FAN Difeng, SUN Yi, MAO Yalang, et al. Analysis of medium motions under the hybrid motion state in the ball mill[J]. Mining \& Processing Equipment, 2010, 38(5): 81-83.

[16] POWELl M S, MCBRIDE A T. A three-dimensional analysis of media motion and grinding regions in mills $[\mathrm{J}]$. Minerals Engineering, 2004, 17(11-12): 1099-1109.

[17] MCBRIDE A, GOVENDER I, POWELL M S, et al. Contributions to the experimental validation of the discrete element method applied to tumbling mills[J]. Engineering Computer International Journal of Computer-Aided Engineering, 2004， 21(2): 119-136.

[18] GOVENDER I, POWELL M S, NURICK G N, et al. 3D particle tracking in a mill: A rigorous technology for verifying DEM predictions[J]. Mineral Engineering, 2001, 10(14): 1329-1340.

[19] FRANKE J, CLEARY P W, SINNOTT M D. How to account for operating condition variability when predicting liner operating life with DEM-A case study[J]. Minerals Engineering, 2015， 73(3): 53-68.

[20] CLEARY P W, OWEN P. Effect of liner design on performance of a $\mathrm{HICOM}^{\circledR}$ mill over the predicted liner life cycle[J]. Minerals Engineering, 2015, 134(11): 11-22.

[21] LU G, THIRD J R, MÜLLER C R. Discrete element models for non-spherical particle systems : From theoretical developments to applications[J]. Chemical Engineering Sciences, 2015，127(12): 425-465.

[22] PAUL W, CLEARY P W, ROB D. Understanding fine ore breakage in a laboratory scale ball mill using DEM[J]. 
Minerals Engineering, 2011, 24(3-4): 352-366.

[23] ZHONG W Q, YU A B, LIU X J, et al. DEM/CFD-DEM modeling of non-spherical particulate systems: Theoretical developments and applications[J]. Powder Technology, 2016, 302(11): 108-152.

[24] 唐果宁, 彭猛. 离散磨介群动力学特性数值分析[J]. 振 动与冲击, 2009, 28(8): 184-187.

TANG Guoning, PENG Meng. Numerical analysis of dynamic characteristics of discrete grinding media[J]. Journal of Vibration and Shock, 2009， 28(8): 184-187.

[25] 唐果宁, 彭猛. 振动磨机离散磨介群动力学模型研究 [J]. 中国机械工程, 2008, 19(11): 1347-1350.

TANG Guoning, PENG Meng. Research on dynamics model of disperse mill medium group for vibration mill[J]. China Mechanical Engineering, 2008, 19(11): 1347-1350.

[26] SUN Y, HUANG X H, JIN X H. Numerical analysis of the ball mill coupled with the vertical vibration frontiers of energy, materials and information engineering[C]// Switzerland: Advanced Materials Research, 2014, 1044-1045: 638-642.

[27] SUN Y, LIANG M, JIN X H, et al. Experimental and modeling study of the regular polygon angle-spiral liner in ball mills[J]. Chinese Journal of Mechanical Engineering, 2017, 30(2): 363-372.

[28] 梁曼. 球磨过程介质群运动区域特性对物料破碎效率 的影响机理研究[D]. 杭州: 浙江工业大学, 2017.

LIANG Man. Influence mechanism of region-character of media motion on grinding efficiency of particles in ball
milling[D]. Hangzhou: Zhejiang University of Technology, 2017.

[29] 应男. 颤振球磨机的介质碰撞能量与粉碎性能的研究 [D]. 杭州: 浙江工业大学, 2012.

YING Nan. Research on charge collision energy and grinding performance of flutter ball mill[D]. Hangzhou: Zhejiang University of Technology, 2012.

[30] 梁曼, 孙毅, 单继宏, 等. 颤振球磨机中颗粒群粒度特 性影响因素试验研究[J]. 机械工程学报, 2018, 54(7): 205-215.

LIANG Man, SUN Yi, SHAN Jihong, et al. Experiment on the influence of features of the broken particle size in a flutter ball mill[J]. Chinese Journal of Mechanical Engineering, 2018, 54(7): 205-215.

[31] 毛亚郎, 孙毅, 计时鸣, 等. 落球冲击破碎下的非限制 料层夹持形态 [J]. 中国机械工程, 2016, 27(2): 168-172. MAO Yalang, SUN Yi, JI Shiming, et al. Nipped configuration of unconfined particle beds under falling ball impact test $[\mathrm{J}]$. Chinese Journal of Mechanical Engineering, 2016, 27(2): 168-172.

[32] DENIZ V. A study on the specific rate of breakage of cement materials in a laboratory ball mill[J]. Cement and Concrete Research, 2003, 33: 439-445.

作者简介: 梁曼, 女, 1991 年出生, 博士, 讲师。主要研究方向为机械 集成系统设计、制造，生丝摩擦、检测技术。

E-mail: 18a0104152@cjlu.edu.cn

孙毅(通信作者), 男, 1966 年出生, 博士, 教授, 博士研究生导师。主 要研究方向为制造业信息化和机电装备设计。

E-mail: sunyi@zjut.edu.cn 